\title{
The EuroSciCon's 2015 Innate Immunity Summit
}

\author{
Marta Camprubí-Rimblas*, ${ }^{*}$, Francesco Peri ${ }^{2}$, Áine McKnight ${ }^{3}$, \\ Claudia Matteucci ${ }^{4} \&$ Raquel Guillamat-Prats ${ }^{1,5}$
}

\section{The EuroSciCon's 2015 Innate Immunity Summit, London, UK, 17-19 November 2015}

\begin{abstract}
A first line of defense against viral infection is prompted by the innate immune system. Viruses activate both extracellular and intracellular events that lead to a war between the virus and the host. In addition to vaccines which induce adaptive T- and B-cell response in readiness for infection, other therapies that potentiate the host immune response are in development, such as those that induce an increase in restriction factor activity or diminish inflammation through Toll-like receptors' antagonists. Other modulators of immune response, such as thymosin $\alpha-1$, contribute to the inhibition of HIV-1 and human T lymphotropic virus 1 infection. Understanding the mechanisms by which the innate immune response combats pathogen invasion will enable the generation of novel therapeutic strategies to cure viral infection.
\end{abstract}

First draft submitted: 10 August 2016; Accepted for publication: 2 September 2016;

Published online: 28 September 2016

Viruses are intracellular parasites that replicate inside host living cells; bacteria, archaea and eukaryotic cells [1]. When entering a host, viruses stimulate different responses and effector mechanisms of the immune system [2]. The ability of the parasite to evade or fight host responses would compromise the survival and pathogeny of the virus.

The host has, and imposes, multiple obstacles to virus infection. The immune system is composed of different defense mechanisms that avoid virus entry, maintain integrity, homeostasis and survival of the host. The first line of defense to the exposure of a new pathogen is initiated by the innate immune system [3-5]. This is based on membrane receptors and phagocytic cells that protect the host from infection. Receptors recognize conserved features of virus and promote a rapid response [6].

There are barriers in vivo that comprise the initial defense against viral infection [7]. Epithelia and mucosal epithelia provide a physical barrier against pathogen entry [8,9]. Mucus secreted by mucosal epithelia traps parasites, while tears and urine are ways to eliminate pathogens. Chemical barriers, such as digestive enzymes or bile salts in the upper GI tract, acid $\mathrm{pH}$ of the stomach or pulmonary surfactant at the lungs, also contribute to killing of pathogens.

'Fundació Parc Taulí, Parc Taulí, Sabadell, Spain

${ }^{2}$ Department of Biotechnology \& Biosciences, University of Milano-Bicocca, Milano, Italy

${ }^{3}$ Centre for Immunology \& Infectious Disease, Blizard Institute, Barts \& The London School of Medicine \& Dentistry, Queen Mary

University of London, London, UK

${ }^{4}$ Department of Experimental Medicine \& Surgery, University of Rome 'Tor Vergata,' Via Montpellier, 100133 Rome, Italy

${ }^{5}$ CIBERES Enfermedades Respiratorias, Parc Taulí, Sabadell, Spain

*Author for correspondence: mcamprubi@tauli.cat \section{Y}


However, once these barriers have been breached, host macrophages play a key role in eliminating parasites. Macrophages are immunological cells that have the ability to phagocytose pathogens and release mediators to recruit neutrophils, NK cells and lymphoid cells at the site of infection. Every organ of the body presents tissue macrophages, which are tailored to the requirements of the tissue and provide a first and quick line of defense. Adaptive immunity provides specificity and memory, and this increases the kinetics of specific responses upon re-exposure to the same parasite [10].

Mills et al. proposed a classification of macrophages phenotype in M1/M2, which determine T-cell response [11,12]. On the one hand, M1 macrophages stimulate the release of cytotoxic Th1 cytokines, such as IFN- $\gamma$ and TNF- $\alpha$ by $\mathrm{T}$ cells, increasing M1 macrophages activation and promoting pathogens destruction. On the other hand, M2 macrophages promote the production of antibody-mediated Th2 cytokines like IL- 4 and IL-10 by T cells. The expression of these cytokines leads to B-cell proliferation, antibody production and an increase of M2 response; maintaining the integrity of the tissue. Furthermore, NK cells can either produce IFN- $\gamma$ to stimulate M1 macrophages or induce cytolysis to viral-infected cells. Most antiviral vaccines [13] are based on the stimulation of B- and T-cell response, in order to induce the basic goals of adaptive immunity, specificity and memory, to improve immunity to a particular pathogen. Vaccines are composed of inactivated or attenuated viruses or products derived from them to stimulate the immune system in order to facilitate the recognition and killing of that particular parasite in future encounters. The identification of new critical antigenic sites and the development of novel delivery systems of antiviral vaccines may offer solutions for vaccines against viruses that currently have no effect.

After the virus has overcome the primary defense of the host and reached the target cell, the attachment phase takes place. First, viral membrane attaches to the host cell surface, in a nonspecific manner, in order to produce an initial interaction to facilitate the binding of viral surface proteins and their specific receptors on the host cellular surface [14]. Once bound to specific cell surface receptors, viruses fuse and enter inside the host cell. Receptors can target virus for endocytosis or trigger signaling pathways for the entry of the pathogen or drive fusion/penetration of the virus at the cell membrane or endocytic compartments [14]. HIV reaches the intracellular space through the fusion of the viral and cellular membranes [15].

After that, the viral genomic RNA is reverse transcribed to DNA. The sequence of events to obtain DNA is vulnerable to the cells' innate restriction factors [16]. Restriction factors are germline-encoded proteins produced by all cells and are not exclusive to immune cells types. Restriction factors are constitutively expressed but can be activated or upregulated by interferon (IFN), such as SAMHD1 and members of the apolipoprotein B mRNA-editing enzyme, catalytic polypeptide-like (APOBEC) family [17], which have been proved to work at the initial stage of reverse transcription of HIV-1. SAMHD1 is expressed in macrophages, dendritic cells and $\mathrm{CD}^{+}{ }^{+} \mathrm{T}$ cells, and degrades the deoxyribonucleoside triphosphates (dNTPs) required for efficient reverse transcription. Members of the APOBEC cytidine deaminases family catalyze the deamination of cytidine to uridine in the nascent DNA [18]. Furthermore, McKnight and coworkers studied other restriction factors for HIV, such as RPRD2 or RNA-associated early-stage antiviral factor (REAF) [17]. REAF is apparently not upregulated by INF- $\alpha$ and is constitutively expressed in human macrophages and $\mathrm{CD}^{+} \mathrm{T}$ cells. REAF is a potent inhibitor of HIV replication. Unfortunately, viruses have evolved to escape from restriction factors [19]. Viral proteins Vpx and Vif mitigate SAMHD1 or APOBEC, respectively, although the antagonist for REAF has not been identified [17].

\section{Host antiviral defense}

In order to combat viral infection, antiviral defense mechanisms take place. Viral coat proteins and viral nucleic acids stimulate pattern recognition receptor, such as Toll-like receptor (TLR) signaling $[4,20]$. Members of TLR1, TLR2, TLR3, TLR4, TLR6, TLR7, TLR8 and TLR9 have been proved to be involved in response to viral infection [21]. For example, DNA viruses, such as human cytomegalovirus, HSV, EBV and vaccinia virus (VACV), and RNA viruses, such as lymphocytic choriomeningitis virus, HCV and respiratory syncytial virus, release pathogen-associated molecular patterns (PAMPs) that stimulate TLR2 triggering an immune response. Protein-based viral PAMPs stimulate TLR2 while viral nucleic acids stimulate TLR3-, TLR8- and TLR9-mediated 
response [22]. Some viruses, such as HCV, take advantage of the innate immune system. HCV core proteins stimulate TLR2 from monocytes, inducing IL-10 and TNF- $\alpha$ cytokines, that would increase dendritic cells' apoptosis and diminish IFN- $\alpha$ release, leading to a diminution of M2 macrophages' activation [23]. All that will favor the evasion of virus from the immune system.

Further, damage-associated molecular patterns (DAMPs) released as a consequence of an infection and derived from damaged, necrotic or infected tissues, can also activate these receptors. Multiple lung insults by chemical (e.g., acid) and diverse microbial agents (e.g., influenza viruses and SARS) cause high lethality due to acute lung injury (ALI) [24]. Imai et al. suggested that such insults trigger reactive oxygen species generating host-derived oxidized phospholipid (DAMPs) that, in turn, stimulates a common TLR4-, TIR-domain-containing adapter-inducing IFN- $\beta$ (TRIF-), and IL-6-dependent pathway in macrophages leading to ALI [24]. Therapeutic approaches work on TLR 4 inhibition by specific antagonists with a chemical structure similar to that of natural lipid A. Despite the potent antisepsis activity of eritoran in animal sepsis models, and despite its excellent safety profile in humans, it failed in a Phase III randomized controlled trial for all causes of sepsis [25]. Treatment of influenza virus-infected mice with eritoran blocked influenza virus-induced lethality and ALI [26]. The mechanism of action of eritoran is suspected to be based on the inhibition of TLR4 activation by endogenous DAMPs produced in the late phase of viral infection [26]. Moreover, several generations of synthetic glycolipids have been developed, which similarly to eritoran, mimic the lipid A moiety of lipopolysaccharide (LPS) and are potent and selective TLR4 antagonists [27-29]. These molecules antagonize TLR4 by interacting with its coreceptors CD14 and MD-2 [30]. One of these molecules, named IAXO-102, was recently found to suppress experimental abdominal aortic aneurysm by its action on TLR4, suggesting that it is a potential drug to treat aneurysms [31]. Another synthetic glycolipid, named FP7 [29], protected mice from influenza virus-induced lethality and reduced both proinflammatory cytokine gene expression in the lungs and ALI [Peri F, Unpublished Data].

Other antiviral strategies are the initiation of the complement system by viruses [32]. On the one hand, this system can be activated by classical pathway, induced by pathogens or by antibody binding to the pathogen surface. On the other hand, the complement system can be activated by the lectin pathway, started by collectins and ficolins. Furthermore, the complement system can be activated by the alternative pathway, which amplifies the other pathways [33]. For instance, collectins and ficolins are pattern recognition molecules that recognize PAMPs. These molecules have pro- and anti-inflammatory activities on the immune system and activate complement through their associated mannose binding lectin associated serine proteases (MASPs). Furthermore, there exist other collectin members such as surfactant proteins A and D which do not work through complement, as they do not activate MASPs. Surfactant proteins $\mathrm{A}$ and $\mathrm{D}$ are found on mucosal surface, and they make pathogens more vulnerable to macrophages' phagocytosis. The three pathways of complement converge on $\mathrm{C} 3$ convertase generation [32], leading to the activation of the complement system. This activation induces the lysis of viruses and infected cells, or targets them for destruction by innate immune cells. Furthermore, the complement system stimulates cytokine production to enhance antiviral response.

\section{Modulators of antiviral response}

Nowadays, there are some specific drugs against viral replication. Thymosin alpha-1 (T $\alpha 1)$ is a thymic-derived peptide that has been used in infectious disease, cancer, immune defenses and inflammation, especially in combination with other drugs. This peptide plays a specific role in lymphoid cells [34]. Matteucci et al. proved that in lipopolysaccharide stimulated $\mathrm{CD}^{+}$ cells, $\mathrm{T} \alpha 1$ potentiates the release of soluble factors able to inhibit HIV-1 and HTLV-1 infection in peripheral blood mononuclear cells [35]. Further studies on T $\alpha 1$ administration together with new treatments and vaccines would be of interest for the treatment of HIV-1 and HTLV-1 infection.

\section{Conclusion \& future perspective}

Although several studies have been performed about viruses' cycle into the host living cells and the host immunological response generated, there are still many unknown mechanisms to be uncovered. Therapeutic approaches based on the stimulation of factors that would act on the extracellular or intracellular viral phase 
are ongoing. Antiviral agents work on different stages of virus development, such as entrance or uncoating inhibitors, or potentiate restriction factors action. Furthermore, inactivated or attenuated vaccines stimulate the immune system to identify and kill pathogens in later infections. Although some viral therapeutic approaches are very potent, many viral diseases are still of significant impact on public health population. Moreover, we should never forget the constant evolution of viruses, which is faster compared with host cells, giving them new methods to escape from the host immune system. Additionally, to improve our knowledge about virus development, it is important to learn more about host immune response, in order to facilitate virus elimination. Molecules able to modulate immune response and to counteract infections, such as $T \alpha 1$, combined with other new treatments or vaccines would improve their effect and enhance the development of potent therapeutic drugs.

\section{Financial \& competing interests disclosure}

The authors would like to thank Innate Immunity Summit EuroSciCon for supporting this article. The authors have no other relevant affiliations or financial involvement with any organization or entity with a financial interest in or financial conflict with the subject matter or materials discussed in the manuscript apart from those disclosed.

No writing assistance was utilized in the production of this manuscript.

\section{EXECUTIVE SUMMARY}

\section{Host immune system}

- Physical and chemical barriers.

- Macrophages:

- $\quad$ M1: stimulates Th1 cytokines inducing pathogen destruction.

- M2: stimulates Th2 cytokines stimulating B-cell proliferation and antibody production.

- Vaccines stimulate B- and T-cell response to facilitate the recognition and killing of the particular parasite.

- Virus cell cycle: restriction factors generated by the host cell:

- $\quad$ SAMHD1 and members of the apolipoprotein B mRNA-editing enzyme, catalytic polypeptide-like family, induced by IFN- $\gamma$.

- $\quad$ RNA-associated early-stage antiviral factor independent of IFN- $\gamma$.

- Toll-like receptors, stimulated by viral proteins:

- IAXO-102 antagonizes TLR4 and inhibits aortic aneurysms.

- Complement system:

- $\quad$ Lectin pathway: activated by collectins and ficolins.

- Classical pathway stimulated by pathogens or antibodies binding to the pathogen surface.

- $\quad$ Alternative pathway: amplifies the other pathways.

\section{Modulators of antiviral response}

- Thymosin $\alpha-1$ : increases the release of soluble factors that inhibit HIV-1 and HTLV-1.

\section{References}

Papers of special note have been highlighted as:

- of interest; $\bullet \bullet$ of considerable interest

1 Buchmann K. Evolution of innate: clues from invertebrates via fish to mammals. Front. Immunol. 5, 459 (2014).

2 Koyama S, Ishii KJ, Coban C, Akira S. Innate immune response to viral infection. Cytokine 43(3), 336-341 (2008).

- Summarizes the roles of pattern recognition receptors and their pathways.
Hato T, Dagher PC. How the innate immune system senses trouble and causes trouble. Clin. J. Am. Soc. Nephrol. 10(8), 1459-1469 (2015).

4 Kondili M, Roux M, Vabret N, Bailly-Bechet $M$. Innate immune system activation by viral RNA: how to predict it? Virology 488, 169-178 (2016).

5 Mansur DS, Smith GL, Ferguson BJ. Intracellular sensing of viral DNA by the innate immune system. Microbes Infect. 16(12), 1002-1012 (2014).
6 Lewis SH, Obbard DJ. Recent insights into the evolution of innate viral sensing in animals. Curr. Opin. Microbiol. 20, 170-175 (2014).

7 Janeway CA Jr, Travers P, Walport M et al. Immunobiology: the Immune System in Health and Disease (5th Edition). Garland Science, New York, NY, USA (2001).

8 Skabytska Y, Kaesler S, Volz T, Biedermann T. How the innate immune system trains immunity: lessons from studying atopic dermatitis and cutaneous bacteria. J. Dtsch. Dermatol. Ges. 14(2), 153-156 (2016). 
9 Kawamura T, Ogawa Y, Aoki R, Shimada S. Innate and intrinsic antiviral immunity in skin. J. Dermatol. Sci. 75(3), 159-166 (2014).

10 Mills CD, Ley K. M1 and M2 macrophages: the chicken and the egg of immunity. J. Innate Immun. 6(6), 716-726 (2014).

11 Mills CD. Anatomy of a discovery: M1 and M2 macrophages. Front. Immunol. 6, 212 (2015).

12 Mills CD, Ley K, Buchmann K, Canton J. Sequential immune responses: the weapons of immunity. J. Innate Immun. 7(5), 443-449 (2015).

- Review of the different ways of immune responses' activation.

13 Graham BS. Advances in antiviral vaccine development. Immunol. Rev. 255(1), 230-242 (2013).

- New critical antigenic sites and novel delivery systems of viral vaccines.

14 Grove J, Marsh M. The cell biology of receptor-mediated virus entry. J. Cell Biol. 195 (7), 1071-1082 (2011).

15 Engelman A, Cherepanov P. The structural biology of HIV-1: mechanistic and therapeutic insights. Nat. Rev. Microbiol. 10 (4), 279-290 (2012).

16 Duggal NK, Emerman M. Evolutionary conflicts between viruses and restriction factors shape immunity. Nat. Rev. Immunol. 12(10), 687-695 (2012).

17 Marno KM, Ogunkolade BW, Pade C, Oliveira NM, O'Sullivan E, McKnight Á. Novel restriction factor RNA-associated early-stage anti-viral factor (REAF) inhibits human and simian immunodeficiency viruses. Retrovirology 11, 3 (2014).

-• Demonstrates the potential effect of early-stage antiviral factor as HIV inhibitor, acting at the early replication cycle of HIV.
18 Wolf D, Goff SP. Host restriction factors blocking retroviral replication. Annu. Rev. Genet. 42, 143-163 (2008).

19 Duggal NK, Emerman M. Evolutionary conflicts between viruses and restriction factors shape immunity. Nat. Rev. Immunol. 12(10), 687-695 (2012).

20 Iwasaki A, Medzhitov R. Control of adaptive immunity by the innate immune system. Nat. Immunol. 16(4), 343-353 (2015).

21 Carty M, Bowie AG. Recent insights into the role of Toll-like receptors in viral infection. Clin. Exp. Immunol. 161(3), 397-406 (2010).

22 Sparrer KM, Gack MU. Intracellular detection of viral nucleic acids. Curr. Opin. Microbiol. 26, 1-9 (2015).

23 Dolganiuc A, Chang S, Kodys K et al. Hepatitis $\mathrm{C}$ virus (HCV) core proteininduced, monocyte-mediated mechanisms of reduced IFN-alpha and plasmacytoid dendritic cell loss in chronic HCV infection. J. Immunol. 177, 6758-6768 (2006).

24 Imai Y, Kuba K, Neely GG et al. Identification of oxidative stress and Toll-like receptor 4 signaling as a key pathway of acute lung injury. Cell 133, 235-249 (2008).

25 Opal SM, Laterre PF, Francois B et al. Effect of eritoran, an antagonist of MD2-TLR4, on mortality in patients with severe sepsis: the ACCESS randomized trial. JAMA 309, 1154-1162 (2013).

26 Shirey KA, Lai W, Scott AJ et al. The TLR4 antagonist eritoran protects mice from lethal influenza infection. Nature 497, 498-502 (2013).

27 Piazza M, Rossini C, Della Fiorentina S et al. Glycolipids and benzylammonium lipids as novel anti-sepsis agents: synthesis and biological characterization. J. Med. Chem. 52(4), 1209-1213 (2009).

Rodriguez Lavado J, Sestito SE, Cighetti R et al. Trehalose- and glucose-derived glycoamphiphiles: small-molecule and nanoparticle Toll-Like receptor 4 (TLR4) modulators. J. Med. Chem. 57, 9105-9123 (2014).

29 Cighetti R, Ciaramelli C, Sestito SE et al. Modulation of CD14 and TLR4.MD-2 activities by a synthetic lipid A mimetic. ChemBioChem. 15(2), 250-258 (2014).

30 Piazza M, Yu L, Teghanemt A, Gioannini T, Weiss J, Peri F. Evidence of a specific interaction between new synthetic antisepsis agents and CD14. Biochemistry 48, 12337-12344 (2009).

31 Huggins C, Pearce S, Peri F, Neumann F, Cockerill G, Pirianov G. A novel small molecule TLR4 antagonist (IAXO-102) negatively regulates non-hematopoietic Toll like receptor 4 signalling and inhibits aortic aneurysms development. Atherosclerosis 242(2), 563-570 (2015).

-• Shows the antagonist effect of IAXO-102 on TLR 4 and the inhibition of aortic aneurysm development.

32 Stoermer KA, Morrison TE. Complement and viral pathogenesis. Virology 411(2), 362-373 (2011).

33 Degn SE, Thiel S. Humoral pattern recognition and the complement system. Scand. J. Immunol. 78(2), 181-193 (2013).

34 Matteucci C, Minutolo A, SinibaldiVallebona P et al. Transcription profile of human lymphocytes following in vitro treatment with thymosin alpha-1. Ann. N. Y. Acad. Sci. 1194, 6-19 (2010).

35 Matteucci C, Minutolo A, Pollicita M et al. Thymosin $\alpha 1$ potentiates the release by CD8 $\left(^{+}\right)$cells of soluble factors able to inhibit HIV-1 and human T lymphotropic virus 1 infection in vitro. Expert Opin. Biol. Ther. 15(Suppl. 1), S83-S100 (2015).

-. Indicates the potential effect of thymosin $\alpha-1$ to inhibit HIV-1 and HTLV-1. 